\title{
Redes interconectadas de docentes: Asimilación trascendente de colaboración pedagógica
}

Ana Aurora Zavala Morales. ${ }^{1}$, Isabel Marina Anchundia Delgado. ${ }^{2}$, Mariana de Lourdes Montenegro Parrales. ${ }^{3}$, Gonzalo Eduardo Zhicay Muy. ${ }^{4}$ \& Jennifer Dolores Anchundia Párraga. ${ }^{5}$

\begin{abstract}
DOI: https://doi.org/10.33262/cienciadigital.v3i3.620

This article selected and adjusted part of the information that is processed as advances to a research in process, located in the interpretative qualitative paradigm, transcendental phenomenological type with support in the hermeneutics, proposed to generate a theoretical construct of emergent elements given in the perceptions of the social actors about collaborative learning as a transdisciplinary view in the context of the Eduardo Vásquez Dodero Educational Center of Fe y Alegría, Ecuador. The gathering of the information was gathered with the in-depth interview applied to three teachers from the context of the study, so that they could contribute their experiences and experiences in the reality of the phenomenon. The categories were selected as: technological teachers' networks, virtual transcendent collaboration and pedagogical situation mediated by ICT, from which the subcategories emerged: collaboration of the groups through technology, sensitivity of the teacher in the common work, integral impact flexible activities, improvement of pedagogical practices, technological experiences available to social service, synchronous and asynchronous times of activities and holistic technological pedagogy, which are integrated to give meaning and meaning to the situation. It is suggested to assume the potential of selflearning, communication and updating offered by ICT, against the commitment of continuous professional improvement of the teacher, which implies institutional support of infrastructure, equipment and Internet connectivity available to promote the development of this collaborative profile of interconnected networks of teachers focused on the pedagogy addressed in these times of the digital age.
\end{abstract}

Keywords: Interconnected Networks Of Teachers, Transcendental Pedagogical Training, Collaborative Learning.

\footnotetext{
${ }^{1}$ Escuela "Eduardo Vásquez Dodero" Fe y Alegría, Ecuador, auroritazavala@yahoo.es

${ }^{2}$ Unidad Educativa Galileo Galilei, Ecuador, isabelanchundia@ hotmail.com

${ }^{3}$ Unidad Educativa Juan Dagoberto Montenegro Rodríguez, Ecuador, mayimp23@ hotmail.com

${ }^{4}$ Escuela Egb Ciudad de Gualaceo, Ecuador, eduzhim@ @otmail.com.

${ }^{5}$ Unidad Educativa Costa Azul, Ecuador, jenniferanchundia1988@gmail.com
} 


\section{RESUMEN}

Este artículo seleccionó y ajustó parte de la información que se procesa como adelantos a una investigación en proceso, ubicada en el paradigma cualitativo interpretativo, de tipo fenomenológico trascendental con apoyo en la hermenéutica, planteada para generar un constructo teorético de elementos emergentes dados en las percepciones de los actores sociales acerca del aprendizaje colaborativo como una mirada transdisciplinaria en el contexto del Centro Educativo Eduardo Vásquez Dodero de Fe y Alegría, Ecuador. El acopio de la información se recabó con la entrevista a profundidad aplicada a tres docentes del contexto del estudio, para que aportaran sus vivencias y experiencias en la realidad del fenómeno. Las categorías se seleccionaron como: redes tecnológicas de docentes, colaboración trascendente virtual y situación pedagógica mediada por TIC, a partir de las cuales emergieron las subcategorías: colaboración de los grupos a través de la tecnología, sensibilidad del docente en el trabajo común, impacto integral flexible de las actividades, mejora de las prácticas pedagógicas, experiencias tecnológicas dispuestas al servicio social, tiempos sincrónicos y asincrónicos de las actividades y pedagogía tecnológica holística, que se integran para otorgar sentido y significado a la situación planteada. Se sugiere asumir el potencial de autoaprendizajes, comunicación y actualización que ofrecen las TIC, frente al compromiso del mejoramiento profesional continuo del docente, lo cual implica apoyo institucional de infraestructura, equipos y conectividad a Internet disponibles para que se promueva el desarrollo de este perfil colaborativo de redes interconectadas de docentes centrados en la pedagogía abordada en estos tiempos de la era digital.

Palabras clave: Redes Interconectadas De Docentes, Formación Pedagógica Trascendente, Aprendizaje Colaborativo.

\section{Introducción}

La búsqueda constante y apegada a las mejores prácticas pedagógicas, son parte de la alineación que se ha de sostener en la aplicabilidad de las políticas públicas educativas que buscan la optimización de las estrategias y situaciones en la construcción del conocimiento, como reto superable, tanto en las condiciones de tiempo como espacios disponibles, dado la dinámica interactiva que exige enfoques y precisiones del docente para indagar, a través de la red, los perfiles significativos que se añaden a sus competencias tecnológicas, al conformar comunidades de aprendizaje valorados en colaboración con los otros. Así, el impacto de las TIC en este referente de fuerza apalanca la actualización permanente y experiencias auto-formativas en el desempeño que va más allá de las promesas de distintos programas de capacitación del docente, pus asumen niveles de realidad concretas en el haber cognitivo tecnológico.

Tales implicaciones involucradas en la sociedad en red, mantienen coincidencia con los razonamientos de Mominó de la Iglesia \& Sigalés (2017) al significar la trascendencia en el foco 
de la acción educativa con énfasis en la individualización del aprendizaje y en la estructuración legible de adaptación a las necesidades e intereses diversos que avanzan progresivamente en la colaboración tecnológica más allá de la configuración del espacio y el tiempo escolar, como respuesta a la multireferencialidad de escenarios y concepciones versátiles, intelectivas y trascendentes por la adaptación del potencial funcional alternativo que ofrecen identificadas con las tecnologías de la información y comunicación (TIC), al expandir los contextos y situaciones que van más allá de las instituciones educativas y pueden satisfacer las nuevas exigencias pedagógicas intercambiables y abiertas a la colaboración en red.

Por lo tanto, estos argumentos que comparto como investigadora y docente convencida de las nuevas coordenadas que demanda la sociedad del conocimiento en los campos específicos de la pedagogía, hace ver la necesidad de rupturas epistemológicas frente a los dilemas que recombinan la fortaleza de utilización de los dispositivos móviles asociado a los elementos entrelazados en las necesidades de información y comunicación en el sistema de relaciones que se dinamizan desde la cohesión socio educativa y hacia el desafío que domina un mundo cada vez más globalizado y cargado de información, prácticas integrales, experiencias curriculares exitosas y entornos asimilables en la plataforma tecnológica fundamentadas en la creatividad que pueden ayudar como espacio previo para construir conocimiento pedagógico autoformativo.

El referente así dispuesto, en conformidad con Vartiainen, Nissinen, Pöllänen \& Vanninen (2018) destaca la participación impulsada por el interés en comunidades generativas o formas de organización social, en sesiones de diálogos a través de multiópticas de instrumentos electrónicos, diferentes intereses e interrogantes perceptibles desarrolladas mediante el uso de las TIC. Cuestión que da lugar a la red colaborativa tanto, en estudiantes como en docentes, investigadores y expertos invitados para nuevos diseños pedagógicos colaborativos. Este escenario, lo complementa Weitze (2016) quien añade que el aprendizaje en un nuevo entorno híbrido sincrónico desarrolla continuas competencias en los docentes para innovar los diseños de prácticas tecnológicas cultivadas en la educación secundaria superior, al establecer equipos pedagógicos de docentes como una comunidad profesional que agrega valor en el ejercicio de la invitación a otros grupos de apoyo en la perspectiva de colaboración, pues aumentan los niveles de sofisticación, reflexión y proyección integrada a los beneficios que generan las tic, en los aportes y perspectivas pedagógicas de la comunidad virtual.

Ello, al destacar la importancia de los recursos tecnológicos que han de estar presentes en la administración educativa como elemento fundamental exitoso en la confrontación y complementariedad de razones colaborativas entre pares de docentes que justifican el uso adecuado de la tecnología al servicio de la educación. Situación que es observada por Voogt, Pieters \& Handelzalts (2016), en la idea del diseño colaborativo en equipos de docentes que interceptan la adopción del sistema de reformas pedagógicas detrás del currículo fortaleciendo las habilidades en el uso de las nuevas tecnologías conectadas a las múltiples disciplinas del saber, tanto en los niveles 
declarativos, procesales como esquemáticos y estratégicos que de otro modo, no pudieran acceder a la visualización de manera amigable.

En suma, este artículo como parte de una investigación en proceso orientada para generar un constructo teorético de elementos emergentes dados en las percepciones de los actores sociales acerca del aprendizaje colaborativo como una mirada transdisciplinaria en el contexto del Centro Educativo Eduardo Vásquez Dodero de Fe y Alegría, Ecuador, presenta parte de los resultados y escenarios parciales ubicados como sentidos y significados concebidos en las percepciones de los docentes quienes aportaron sus vivencias y experiencias a través de la entrevista a profundidad. Información que alcanzó su tratamiento en los procesos de categorización y codificación, generando aspectos claves interpretados como: colaboración de los grupos a través de la tecnología, sensibilidad del docente en el trabajo común, impacto integral flexible de las actividades, mejora de las prácticas pedagógicas, experiencias tecnológicas dispuestas al servicio social, entre otras. La estructura teórica comprende las redes: conexiones amigables del docente, formación pedagógica trascendente: colaboración en línea y mejoramiento profesional del docente: oportunidades colaboración en red, las cuales fueron argumentadas y sometida a la hermenéutica de los textos.

\section{Redes pedagógicas: conexiones amigables del docente}

Las bondades que ofrecen las TIC en el referente educacional, son cada vez más utilizables en el núcleo de habilidades tecnopedagógicas cuya importancia atribuida en su condición amigable, respecto al uso integral de los docentes, enfatiza las herramientas socioconstructivas y colaborativas para apropiarse de nuevas realidades paradigmáticas filtradas a través de contenidos digitales e inversión de equipos, motivaciones y sentido de la comunicación en línea, al focalizar el potencial en la vida exitosa profesional del docente. He allí donde White (2014), hace ver que este contexto atribuido a las redes pedagógicas como conexiones amigables del docente, dan cabida a nuevas experiencias prácticas de tipo interactivas que examinan la integración de la tecnología, dada la aceptación y sistema de creencias innovadoras de orientación y colaboración, situaciones integrales de aprendizaje que superan las brechas del sistema de reformas inmersas en la revolución digital, frente al panorama que se valora del Internet para la circulación de la información y el conocimiento y en disponibilidad de soluciones agrupadas en escenarios educativos profesionales, donde los docentes intercambian situaciones pedagógicas de vigilancia tecnológica.

Ahora bien, el profesorado nunca deja de aprender mientras se crean condiciones que les animen al desarrollo profesional respaldado por autoridades institucionales, al otorgar énfasis en la planificación que refleje el desarrollo de las políticas en la gestión de mejoras del desempeño desde la autoevaluación, indagación y fomento de confianza colaborativa, a través de redes creadas en torno a relaciones respetuosas y sentido de apoyo positivo de los maestros para el sistema de mejoras continuas en la práctica pedagógica. Tal como lo mencionan Stoll, Harris \& Handscomb (2012) se trata de aprendizaje en red que ha ganado principios de apoyo ante las evidencias de 
apostar hacia la consecución de los fines educacionales que vinculan el aprendizaje profesional efectivo y prácticas apropiadas de emprendimientos en colaboración, para alentar esfuerzos y explorar el desarrollo profesional que conduzca a la pedagogía activa y constante.

Esta perspectiva, añade valor a la tendencia de superar los problemas y desafíos en las barreras epistemológica que suelen tener algunos docentes para mejorar su desarrollo profesional a través de las TIC confrontando los escenarios de la innovación pedagógica representada por los cursos en línea en términos de motivación, actitudes e integración de actividades de colaboración entre docentes. Al respecto señalan Karsenti, Savoie-zajc \& Iarose (2001), ciertas experiencias de cobertura mediática con el uso de las TIC en la formación práctica y aprendizajes pedagógicos integrados al cambio estructural con estas herramientas que promueven la autonomía, el sentido crítico del profesor en el hecho de enfrentar dilemas y rupturas en las formas de aprender. Situación que da cabida a la tendencia innovadora de recursos en la Web que respalden las estructuras unificadoras y representativas del papel que resaltan la utilidad de las TIC para mejorar la práctica profesional futura de los docentes frente a las facultades críticas, al buscar respuestas a problemas conjuntos y en colaboración con sus iguales.

Es así como los razonamientos de Cleary \& Corti (2008) amplían estas ideas en la tendencia innovadora de formación tecnológica del docente. Sin embargo, la subutilización de los equipos informáticos, las dificultades metodológicas con las cuales se investigan las diferentes áreas y los esfuerzos de tiempo, dedicación y mediación de los docentes, constituyen parámetros de interés para el buen funcionamiento que se espera en los resultados de los aprendizajes, según la disciplina del docente considerado, dadas las orientaciones profesionales, así como los dispositivos de entrenamiento que se mezclan con las múltiples tareas de los profesores, que muchas veces; no coincide de manera coherente e integral con la existencia de los recursos TIC en las instituciones educativas.

Todos estos factores, elementos y condiciones asociados al manejo efectivo de las TIC en el referente de construir redes pedagógicas como conexiones amigables del docente, dan paso abierto a la identificación de las percepciones de los docentes sobre los beneficios reales ofrecidos en la dinámica de su profesión integrada en la colaboración e integración pedagógica, con la posibilidad de cuestionar su propia práctica, frente al apoyo institucional y la autoformación continua que se perfilan en los ejercicios de colaboración al comprender y manejar alternativas dentro de los fenómenos complejos, pertinentes y asimilables de nuevos aprendizajes en la busca de referentes pedagógicos que evidencien los beneficios en el uso de las TIC, dada la capacidad y disponibilidad de los maestros para invertir su tiempo en la integración de estas herramientas, la superación de los obstáculos frente a la insuficiencia del equipamiento tecnológico, la falta constante de la conexión a Internet y la metodología implementada para la integración en la construcción del conocimiento. Algunos de los elementos identificados se visualizan en la figura 1. 
Figura 1. Bondades de las Redes Pedagógicas de Docentes

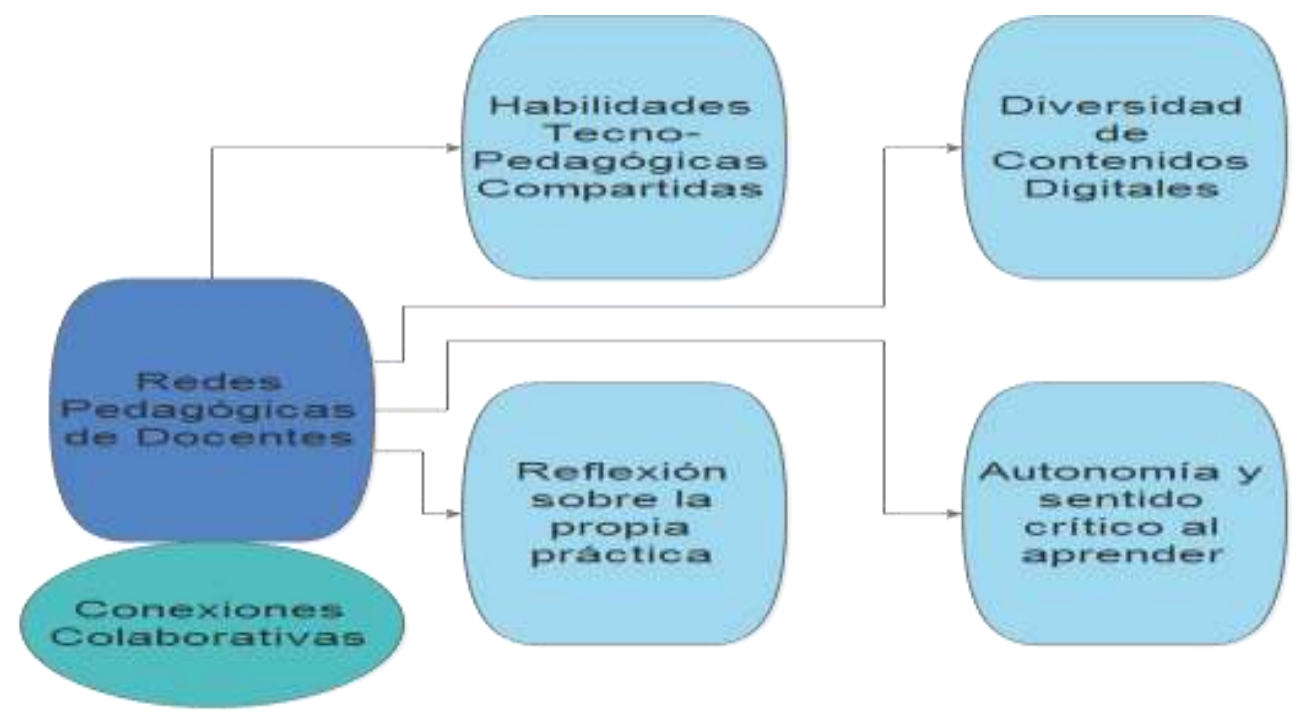

Fuente: Elaboración Propia

La situación que presenta la figura 1, referida a las bondades identificadas en las redes pedagógicas del docente, para el desarrollo e innovación profesional en el sentido de colaboración entre iguales, cobra relevancia en las habilidades tecno-pedagógicas compartidas como decisiones favorables a los nuevos aprendizajes en línea que concretan actividades y procesos más eficaces en el desarrollo de experiencias profesionales al focalizar este compartir del conocimiento pertinente ante la confrontación de alternativas reflexionadas para la solución de problemas complejos en las instituciones educativas, puesto que la integración de competencias, habilidades e intereses, fortalecen el procesamiento en grupo y se trasladan al hecho pedagógico de la cotidianidad institucional. Así también lo manifiestan Montrieux, Vanderlinde, Schellens \& De Marez (2015) ante los cambios que se generan en los elementos percibidos por los docentes cuando transforman los estilos de enseñanza centrados en sí mismos hacia enfoques focalizados en el aprendizaje, cuestión que comparten a través de los diferentes dispositivos interactivos en entornos virtuales, para introducir la innovación técnica y pedagógica que facilita asumir el potencial de beneficios que otorgan las herramientas tecnológicas en el sistema de gestión digital dirigido a la colaboración ante una gama de situaciones pedagógicas integrales que ayudan a la participación de los actores del hecho educativo, en tiempos de transformaciones sociales.

Asimismo, la figura 1, presenta como bondades de la red pedagógica de docentes la generación de diversidad de contenidos digitales, que ayudan, no solamente desde el punto de vista de la madurez de la experiencia significada en el área de meta cognitivo del docente, sino además en los factores percibidos hacia el mejoramiento de la práctica en el escenario institucional; cuestión es resaltada por Barfield (2016) desde la colaboración entre docentes para el aprendizaje cooperativo que 
implica decidir acerca de planificación educativa, desarrollo de objetivos junto a los otros, en el núcleo de la participación de los actores que interactúan en el conjunto de decisiones acerca de los hechos pedagógicos, al tiempo que incluye la dependencia de expertos externos para las diferentes alternativas en la resolución de problemas complejos.

La misma perspectiva, se avizora en la reflexión sobre la propia práctica a la cual hace referencia la figura 1, en correspondencia con los razonamientos de Mathew, Mathew \& Peechattu (2017) como escenario proactivo caracterizado para facilitar los aprendizajes y la comprensión en el desarrollo profesional del docente de manera sistemática y en conformidad con las propias acciones que examinan su ontología al explorar el hecho de estar ahí, en la cotidianidad del ente que orienta sus actividades y procesos, cuando se involucran en la interfaz de la transmisión de conocimientos, habilidades competencias en red de pares, asimilados con la misma tendencia global del hacer continuo en el sistema de mejores formas de concebir el pensamiento educativo. En tales condiciones, entiendo que la interpretación asociada a la acción reflexiva del docente, es propia para considerar la exploración de las tendencias que subyacen a la colaboración de nuevos progresos que ponen énfasis a la enseñanza y aprendizaje reflexivo, dentro de niveles de decisiones entre pares al ayudar efectivamente, a seleccionar ejercicios sensibles y emergentes dada la convicción de utilidad crítica y prácticas de socialización del conocimiento pedagógico en escenarios digitales.

En el mismo orden de ideas, la figura 1, señalan como ámbito de conexión colaborativa entre los docentes el hecho de formarlos en autonomía y sentido crítico al aprender. Así, lo planteado por Asgharheidari \& Tahriri (2015), llama la atención en la capacidad que conforman el desarrollo profesional de los docentes al pensar críticamente como habilidad reflexiva hacia el alcance del éxito académico y social en las tareas y responsabilidades que le son propias en su práctica pedagógica. En este sentido, se comparte con los mencionados autores este traslado de los razonamientos a la condición colaborativa en red entre pares ante la evolución constante de nuevos descubrimientos, que avalan el refuerzo y los condicionantes para tomar de iones en conjunto frente al mundo desafiantes de la tecnología aplicada a la educación. Con ello resalto estos cuatro escenarios importantes para revisar y aprehender las bondades de las redes pedagógicas del docente al interactuar permanentemente para la innovación de sus prácticas, dadas las conexiones colaborativas en conformidad con los aspectos neurálgicos que integran las diversas disciplinas del magisterio, pues exigen de habilidades y competencia metacognitivas en la percepción entre docentes, como actitudes afectivas, críticas y trascendentes para el desarrollo de los propósitos académicos, educativos y profesionales de avanzada, requeridos en la sociedad el conocimiento.

\section{Formación Pedagógica Trascendente: Colaboración en Línea}

La condición trascendental se asocia con la mirada holística del pensamiento y la acción auto formativa del docente con el uso de las TIC para generar espacios de comprensión y colaboración en línea, al fundamentar las competencias consignadas en el interés pedagógico y las áreas 
asociadas a los ejes didácticos, de participación y construcción del conocimiento en red. Sobre esta perspectiva adquiere interés los aportes de Weitze (2017) en la dinámica del Modelo de Think Tank Pedagógico para equipos de profesores, cuya intencionalidad es el desarrollo continuo de competencias colaborativas e innovadoras en el diseño de aprendizajes, dentro de un entorno híbrido sincrónico mediado por videos. En este sendero de prácticas cultivadas se incluyen los marcos sociales, dado la identidad del equipo en una comunidad profesional de prácticas para interactuar. Las palabras del mencionado autor aumentan las oportunidades de construir nuevos diseños pedagógicos donde se pueda apoyar al docente en el marco entendible de sus necesidades de innovación, capacidades tecnológicas y uso efectivo de la Web.

En este sentido, los aportes de la formación pedagógica trascendente también se manifiestan en la perspectiva de Parsons, Thomas, Inkila, Antipas, Valintine \& Pham (2015) enfocad en el aprendizaje y liderazgo digital y colaborativo, en el escenario de prácticas pedagógicas que aseguren el cambio en la realidad de sus efectos, para transformar escuelas a partir de la aplicabilidad de conocimientos relevantes hacia las nuevas generaciones que han crecido con tecnología y mantienen sus expectativas de efectividad en una sociedad más amplia de oportunidades aprovechando las redes sociales. Es así como la construcción del conocimiento exige cambios deliberados en la pedagogía dentro del ciclo de políticas sociales, económicas, tecnológicas y educacionales que avanzan hacia la cultura digital de la educación en constante cambio y evolución.

Los elementos significados por estos autores, que comparto como docente e investigadora, impactan las expectativas de las redes interconectadas para la formación pedagógica trascendente del docente como núcleo significativo de colaboración, al desafiar las sesiones de tiempo, espacios y lugares que adoptan el énfasis en la autogeneración del conocimiento innovador compartido. Tales decisiones, aportan la promoción de la profesionalización del docente interinstitucional para capturar los diferentes tópicos relacionados con la pedagogía que permiten reflexionar acerca de la propia práctica y más allá de ello, en la aplicabilidad digitalizada del aprendizaje colaborativo entre los docentes para el aprovechamiento integral ante los múltiples usos creativos de estrategias, empoderamiento para la resolución de problemas de aprendizaje adoptados y trasladados al ámbito educativo cotidiano cargado de habilidades digitales. La complementariedad sobre estos hechos se respalda en los hallazgos de Green, Wolodko, Stewart, Edwards, Brooks \& Littledyke (2011) en la observación de prácticas pedagógicas que consolidan las experiencias en la autoformación del docente, mediados con el propósito de lograr el aprendizaje profesional a través de la participación y reflexión, implementando la flexibilidad en la transición negociada y participativa dentro de la pedagogía en un entorno en línea.

Todos estos eventos impactan el sentido de la colaboración entre docentes del punto de vista de sus habilidades cognitivas y los niveles del conocimiento que precisan abordajes a distancia a tener en cuenta el referente pedagógico de conceptos y estrategias entre profesores, de acuerdo a las áreas 
de especialización del docente, las actitudes y los contenidos del curso que se construye de manera colaborativa. Tal como lo avalan Lafifi \& Touil (2010) al indagar sobre profesores que no están especializados en TIC pues sólo poseen conocimientos básicos en este escenario y responden con buen impacto en los perfiles cognitivos que aumentan el nivel de conocimientos con la colaboración de sus iguales y desde el seguimiento del motor de búsqueda adecuado para llevar a cabo el aprendizaje conectado a la intranet de la Universidad.

Vale la pena destacar la diversidad de experiencias que exponen lo anteriormente dicho, dado lo popular de los casos que así lo determinan. Por ejemplo, lo mencionado por Islam, Jacks \& Cap (2016) referente a la colaboración en línea acerca de contenidos de las conferencias, como potencial de aprendizajes que combina imágenes y audio, chat para comentarios e interrogantes a través de la plataforma en dispositivos accesibles por la calidad, variedad y funcionabilidad, como tablets o smartphone, para conseguir el feedback apropiado. Situación que conforma un interfaz amigable en el diseño tecnológico para satisfacer las demandas y socialización de los profesores en la capacidad de respuestas efectivas sincrónicas o asincrónicas a las inquietudes.

En suma, la diversidad de criterios de formación pedagógica trascendente como colaboración en línea, constituye una alternativa que se han de examinar para el apoyo efectivo de los equipos de docentes, cuya mirada prospectiva del futuro atañe a la posesión intelectiva de estas formas y escenarios de decisión tecnológica para el aprendizaje y el desarrollo profesional en entornos virtuales que van más allá del intercambio de información, pues se inscriben en hechos colaborativos para construir el conocimiento pedagógico innovador, de beneficios mutuos, de carácter metacognitivo que distingue las comunidades en red, al hacer uso efectivo de las TIC como herramientas que han venido reportando experiencias, factores de apoyo y diseños tecnológicos que impulsan el entendimiento y apego en el avance del docente al adoptar su perfil de competencias con el advenimiento del Internet, los ajustes en la apropiación de los equipos tecnológicos, la disposición y disponibilidad de los tiempos para esta dedicación de iniciativas tendentes a la construcción e interacción social mediada por las TIC.

\section{Mejoramiento Profesional del docente: Oportunidad de Colaboración en Red}

La dinámica profesional del docente, no sólo se debe valorar a través de las experiencias vividas en el ámbito del cumplimiento de sus actividades cotidianas de acuerdo a las diversas responsabilidades que realiza en las instituciones educativas, sino además; ello debe incluir su formación profesional continua, lo cual da cabida al aprovechamiento de prácticas y contactos entre pares, asimilados a través de los recursos tecnológicos, pues éstos ofrecen la oportunidad de mantenerse asesorado en colaboración continua y en correspondencia con los ecos de la innovación tecnológica para el mejoramiento profesional.

En palabras de Mérida, González y Olivares (2017) este contexto tecnológico activa el análisis, la interpretación y reflexión que proporciona realimentación crítica en red, donde pueden intervenir docentes, investigadores y expertos tecnológicos que enriquecen los marcos entendibles del 
desempeño profesional en materia educativa, a partir de las expresiones y asesorías de los mismos profesores que expresan las circunstancias del mejoramiento profesional para superar los dilemas epistemológicos en la dinámica de aprendizajes activos recurrentes, en manifestaciones de confianza en sí mismo, esfuerzos y voluntad motivadora para superar la adversidad. Eventos con los cuales estoy de acuerdo al reconocer los elementos significativos de la capacitación, innovación y transformación que se vincula a las buenas prácticas significadas en redes de docentes en contacto permanente, cuyo incentivo es continuar avanzando en el sistema de mejoras del desempeño al servicio de la educación.

Ahora bien, Ávalos \& Bascope (2017) observan en el escenario del mejoramiento profesional a través de las TIC, las percepciones, creencias y experiencias de los maestros acerca de la colaboración informal que explora la eficacia colectiva dentro de la cultura tecnológica en términos de ayuda mutua para el aprendizaje, la formación permanente, sobre la base de compartir ideas y escriturar opiniones en temáticas educativas puntuales exigentes desde la mirada grupal hacia el servicio educativo en condiciones menos formales y de mayor interacción en las comunidades de práctica profesional en la instancia pedagógica. En tal sentido, los hallazgos de su investigación desplegaron las principales preferencias de los maestros en este tipo de circunstancias que implicaron actividades de reflexión individual basadas en el análisis de casos y con resultados de evaluaciones, de preferencia en las actividades asociadas al trabajo real con colegas, como generar contenidos de textos con colegas o participar aprendizajes de equipos, como también fue significativo el hecho de aprender de la observación de lecciones en conjunto.

La complementariedad acerca de estos hechos se dejan ver en Trusta, Krutkab \& Carpenterc (2016) frente a la popularidad que han ganado en la educación y formación el uso efectivo de las tecnologías al servicio del educador, como ámbito de redes de aprendizaje propios que ayudan al crecimiento del docente, conjuntamente con sus compañeros, por la preferencia de la reducción de tiempos y espacios al responder los diversos intereses y necesidades que posibilitan la construcción del conocimiento pedagógico, de cara hacia el futuro en la era globalizada de la información y la comunicación. A ello se agregan las razones de multiplicidad de dispositivos, que dan cabida a la plataforma digital para auxiliar al perfeccionamiento magisterial, ante manifestaciones flexibles de interacción formal e informal, en diversidad de situaciones que involucran las herramientas tecnológicas de información y comunicación en red.

\section{Materiales y Métodos}

Esta producción académica proviene de los adelantos de una investigación en proceso, de carácter cualitativa, fenomenológica trascendental apoyada en la hermenéutica, cuyo propósito se orientará a generar un constructo teórico en torno a las redes interconectadas de docentes como una asimilación trascendente de colaboración pedagógica. El acopio de la información se obtuvo de la técnica de la entrevista a profundidad, caracterizada por el acercamiento entre iguales, en confianza, cara a cara con el fin de captar la esencia de las conciencias acerca del haber de 
experiencias y vivencias derivadas de las percepciones de tres actores sociales, caracterizados por ser profesores de la educación general básica del Centro Educativo Eduardo Vásquez Dodero, de Ecuador. Estos testimonios fueron transcritos y vaciados en cuadros de contenidos, donde alcanzaron los procesos de categorización y codificación. Las categorías iniciadoras fueron: redes tecnológicas de docentes, colaboración trascendente virtual, y situación pedagógica mediadas por TIC. La codificación de las subcategorías emergentes obedeció a las iniciales de las mismas, el docente que emitió el trozo discursivo y líneas que ubican este significado en el material transcrito de las entrevistas.

\section{Análisis de la Información Empírica y Resultados}

Las condiciones para manejar el material transcrito de las entrevistas en virtud de su análisis e interpretación, no es rígido, no depende de guías para su sistematización, sino que la creatividad del investigador en su abordaje reviste importancia para su presentación y comprensión. Desde este punto de vista, retomamos los argumentos de Strauss y Corbin (2002) para encontrar el sentido a la información recolectada con la modalidad hermenéutica, donde la sensibilidad del sociólogo y su experiencia proporciona ciertas fuentes para enriquecer y estimular el pensamiento en torno a los atributos develados. Así, los autores indican que lo que importa es la percepción de los actores sociales acerca de los acontecimientos o sucesos. "Lo que ayuda es que el investigador tiene una base comparativa contra la cual puede medir el rango de significados y una lista inicial de propiedades y dimensiones dados por los demás" (p. 54). Al considerar estos argumentos, seguí lo propio y derivé el cuadro 1.

Cuadro 1 Categoría: Redes Tecnológicas de Docentes

\begin{tabular}{|c|c|c|}
\hline $\begin{array}{c}\text { Docente } \\
\text { Participante }\end{array}$ & Trozo Discursivo & Subcategoría/Código \\
\hline DA & $\begin{array}{l}\text { Se están dando pasos firmes para asumir esta } \\
\text { condición de grupos apoyados a través de las } \\
\text { tecnologías, pero es un trabajo de cambios de } \\
\text { mentalidad y disposición... }\end{array}$ & $\begin{array}{l}\text { Colaboración de los } \\
\text { grupos a través de la } \\
\text { tecnología, COGTEC, } \\
\text { DA, L: } 17-23\end{array}$ \\
\hline DB & $\begin{array}{l}\text {...el hecho de contar con los equipos, debe ser el } \\
\text { aval para asumir la formación en el manejo de las } \\
\text { TIC, es un trabajo en común y cómo le decía, la } \\
\text { inundación de los dispositivos móviles ha } \\
\text { permitido la sensibilidad de nuestra gente, de } \\
\text { nuestro personal docente. }\end{array}$ & $\begin{array}{l}\text { Sensibilidad del } \\
\text { docente en el trabajo } \\
\text { común, SENDTC, } \\
\text { DB, L: 27-32 }\end{array}$ \\
\hline $\mathrm{DC}$ & $\begin{array}{l}\text {...Se ha creado un clima de sensibilidad por el uso } \\
\text { de las TIC en los docentes en el medio interno para } \\
\text { sentir mayormente la comunicación con los grupos } \\
\text { de apoyo. }\end{array}$ & $\begin{array}{l}\text { Sensibilidad del } \\
\text { docente en el trabajo } \\
\text { común, SENDTC, } \\
\text { DC, L: } 19-23\end{array}$ \\
\hline
\end{tabular}

Fuente: Elaboración Propia 


\section{Hermenéutica Integral de los Textos Descritos en Redes Tecnológicas de Docentes}

La colaboración en red para la formación, actualización y competencias de los docente en el desarrollo de habilidades tecnológicas, cobra vigencia ante la idea de organización pedagógica para la resolución de problemas complejos en la realidad de circunstancias y adaptabilidad de prácticas en el uso de las TIC, que se valora para el aprendizaje educativo ante la sensibilidad que populariza el uso de dispositivos móviles utilizables en el marco común del desempeño docente para mantenerse comunicados y alerta ante las necesidades metacognitivas comunes en el campo trascendente de las nuevas realidades manifestadas en la virtualidad del conocimiento.

Esta perspectiva, se argumenta según Geng, Chai, Jong \& Tsun-Hin Luk (2019), al entender el potencial pedagógico de la interactividad con base a realidades virtuales que dan cabida a la exploración pedagógica en red, sobre la base de la aceptación, creación y experiencia, con fines de producción del conocimiento colaborativo y de cara a las preocupaciones de adopción tecnológica de los docentes, más allá del nivel de aceptación competitivas, acerca de los requisitos, escenarios, infraestructuras y equipos de integración tecnológica a la pedagogía. Cuestión que sugiere, la necesidad institucional de atender con mayor prioridad los esfuerzos para la consecución de prácticas formativas de las capacidades tecnológicas para que el docente pueda diseñar y redefinir la innovación pedagógica en sus desempeños. A continuación el cuadro 2 con la categoría Trascendente Virtual.

Cuadro 2Categoría: Colaboración Trascendente Virtual

\begin{tabular}{|c|c|c|}
\hline $\begin{array}{c}\text { Docente } \\
\text { Participante }\end{array}$ & Trozo Discursivo & Subcategoría/Código \\
\hline $\mathrm{DA}$ & $\begin{array}{l}\text {...Posibilidad de abrir dos acciones pedagógicas en } \\
\text { entornos virtuales y eso se ha de atender desde el } \\
\text { plano operativo anual donde van todas las } \\
\text { actividades de gestión, pedagogía, pastoral y la } \\
\text { parte comunitaria... }\end{array}$ & $\begin{array}{l}\text { Impacto integral } \\
\text { flexible de las } \\
\text { actividades, IMPIFA, } \\
\text { DA, L: } 45-49\end{array}$ \\
\hline DB & $\begin{array}{l}\text { Nosotros ahora estamos en una campaña de } \\
\text { concientización y actualización tecnológica del } \\
\text { docente, por ejemplo, se ha sabido de las } \\
\text { necesidades que salen conjuntamente de todos } \\
\text { ellos diciendo que de verdad lo requieren para } \\
\text { mejorar la situación pedagógica práctica. }\end{array}$ & $\begin{array}{l}\text { Mejora de las } \\
\text { prácticas } \\
\text { pedagógicas, MEPP, } \\
\text { DB, L: 61-64 }\end{array}$ \\
\hline DC & $\begin{array}{l}\text { Yo siempre he dicho: Dios ha sido muy generoso } \\
\text { conmigo por haberme dado la posibilidad de } \\
\text { formarme en la tecnología, no solamente como } \\
\text { profesional, sino también como persona que aporta }\end{array}$ & $\begin{array}{l}\text { Experiencias } \\
\text { tecnológicas } \\
\text { dispuestas al servicio }\end{array}$ \\
\hline
\end{tabular}


parte de las habilidades en este movimiento hacia social, EXPTSS, DC, lo social.

L: $35-39$

Fuente: Elaboración Propia

\section{Hermenéutica Integral de los Textos Descritos en Colaboración Trascendente Virtual}

La integración significativa de las subcategorías emergentes en el discurso de los actores sociales, da cabida a la integración de sus trozos discursivos en razón de la colaboración trascendental virtual que connota la pedagogía más allá del aula, en términos de la comunicación y gestión con el entorno comunitario, frente a lo cual la institución educativa reconoce las necesidades de actuación conjunta, al aportar las experiencias tecnológicas dispuestas al servicio colectivo. En palabras de Espitia \& Olarte (2011), la competencia tecnológica de los profesores se comparte de manera coherente en el marco de las políticas públicas educativas para fomentar la comunicación e interacción con el medio circundante, en términos de discusiones y colaboraciones en experiencias duales a través de la Web, al mostrar informaciones y el despliegue de actividades y procesos con el uso de los foros y los blogs en el desarrollo curricular participativo.

Este escenario da lugar a interpretar la necesidad de comunicación trascendente que ofrecen las TIC para conceder información y oportunidades participativas al conglomerado social que rodea a la institución educativa frente al liderazgo competente del docente al centrar sus esfuerzos en la promoción de prácticas pedagógicas desde la virtualidad y en este fin, conseguir respuestas apropiadas a la conexión social que desarrolla mayor confianza entre los participantes del grupo docente para realizar actividades, estrategias y proyectos que así lo determinen. A continuación el cuadro 3 con la categoría Situación Pedagógica mediadas por TIC.

Cuadro 3 Categoría: Situación Pedagógica Mediadas por TIC

\begin{tabular}{|c|c|c|}
\hline $\begin{array}{c}\text { Docente } \\
\text { Participante }\end{array}$ & Trozo Discursivo & Subcategoría/Código \\
\hline DA & $\begin{array}{l}\text { Fue una opción que nos movió en el ámbito social } \\
\text { del Colegio, pues son pocos los recursos de papel } \\
\text { asignados para ese proyecto, entonces } \\
\text { compartimos un grupo virtual con la gente que en } \\
\text { esos momentos ocupaban lugares distintos en lo } \\
\text { productivo alejados de la comunidad... }\end{array}$ & $\begin{array}{l}\text { Tiempos sincrónicos } \\
\text { y asincrónicos de las } \\
\text { actividades, } \\
\text { SINCASIN, DA, L: } \\
71-78\end{array}$ \\
\hline DB & $\begin{array}{l}\text { S superan las barreras para la colaboración virtual } \\
\text { se incluyen confianza, ante una falta de tiempo, y } \\
\text { una sensación de presión para participar... }\end{array}$ & $\begin{array}{l}\text { Tiempos sincrónicos } \\
\text { y asincrónicos de las } \\
\text { actividades, } \\
\text { SINCASIN, DB, L: } \\
57-61\end{array}$ \\
\hline
\end{tabular}




DC La tecnología ha transformado mi vida para el bien
y eso lo he reflejado, no solamente como docente tecnológica holística,
de fe y alegría, sino como persona en la PEDHOL, DC, L: 83-
comunicación con la familia y en la oportunidad de 89
servir a la comunidad...

Fuente: Elaboración Propia

\section{Hermenéutica Integral de los Textos Descritos en Situación Pedagógica Mediadas por TIC}

Los textos discursivos descritos acerca de la realidad percibida en la situación pedagógica mediadas por TIC, reviste la congruencia interactiva, tanto del ámbito social como personal y pedagógico, cuya plataforma de adaptabilidad es importante de revisar ante las necesidades de comunicación, información y colaboración de la comunidad educativa para superar las deficiencias de tiempos y espacios dedicados a la institución. Estas condiciones de servicio integral mediados por las TIC, se comparte con los planteamientos de Schieffer (2015) cuando los componentes críticos en la línea exitosa, tanto de capacitación en tecnología de los docentes como en el apoyo para satisfacer las necesidades diversas en los requerimientos que se intencionan para la ejecución de proyectos institucionales, subyace al desarrollo profesional y soporte de los docentes al crear actividades de participación remotas para abordar diferentes escenarios. Cuestión que avizoro en términos de las exigencias de la pedagogía tecnológica en las razones de colaboración del profesorado, ante los límites estrechos de recursos administrativos en la gestión escolar.

La siguiente figura 2, refleja la síntesis de los resultados parciales obtenidos en la investigación.

Figura 2. Categorías y sus Subcategorías Emergentes

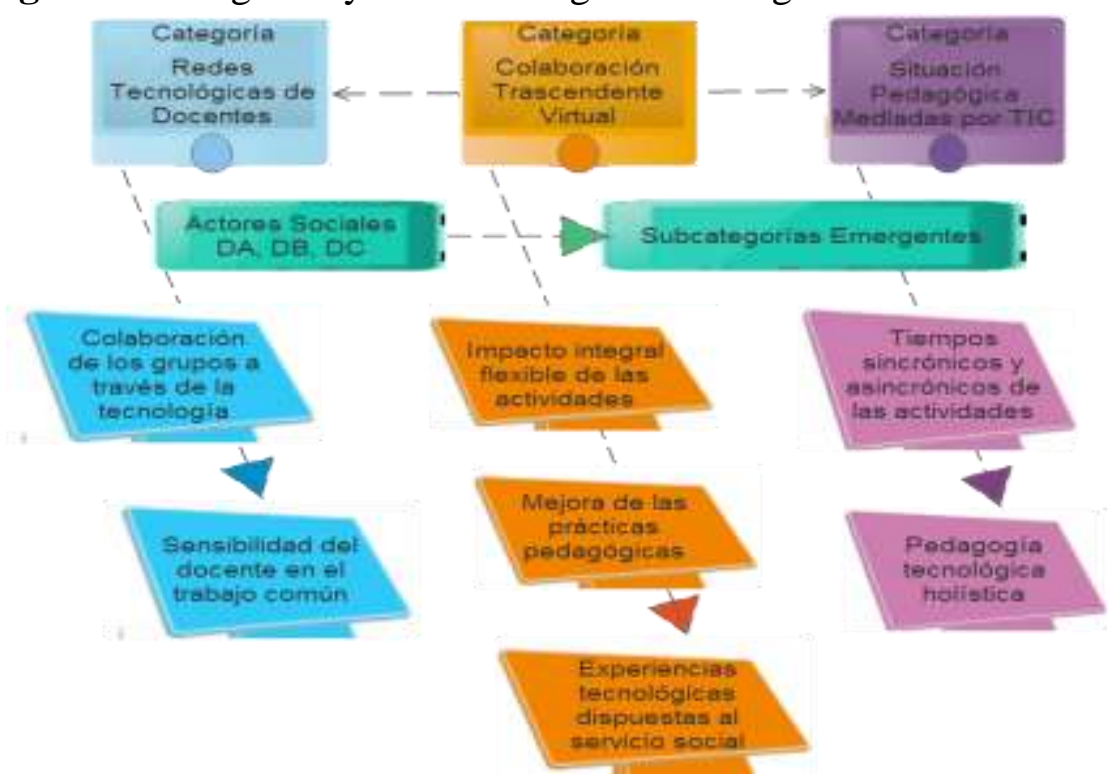

Fuente: Elaboración Propia 
La figura 2, integra las partes en un todo holístico comprensivo develado en las categorías: redes tecnológicas de docentes, colaboración trascendente virtual y situación pedagógica mediadas por TIC, cuyos sentidos y significados se reflejan desde las voces interpretadas de los actores sociales, en la subcategorías emergentes: colaboración de los grupos a través de la tecnología, sensibilidad del docente en el trabajo común, impacto integral flexible de las actividades, mejora de la práctica pedagógica, experiencias tecnológicas dispuestas al servicio social, tiempos sincrónicos y asincrónicos y pedagogía tecnológica holística.

En concordancia con estos hallazgos se comparte con Treharne \& Riggs (2015), el sentido de calidad de la investigación cualitativa, pues resaltan la autenticidad de los sentidos y significados clave para la reflexión de los propios docentes que emitieron sus percepciones, que en este caso, se corresponde con la confianza interna de estas versiones en torno a la realidad del fenómeno de estudio. Asimismo, la reflexibilidad personal que involucró a la investigadora en el perfil de competencias observadas hacia adentro, reflejó los escenarios en las circunstancias informadas e interpretadas en el sentido de las redes interconectadas de docentes como asimilación trascendente de colaboración pedagógica.

\section{Discusión y Presentación de Hallazgos Relevantes}

Los hallazgos derivados en este artículo, cobran vigencia en la sinergia de actuaciones en redes tecnológicas de docentes significadas frente a la colaboración de los grupos a través de la tecnología y la propia sensibilidad del docente en el trabajo común desarrollado en la cotidianidad de sus haceres institucionales. Estas implicaciones coinciden con la investigación de Hennessy, Deaney \& Ruthven (2015) donde destacan las formas del pensamientos diversos (unidad en la diversidad) que se comparten los profesores en prácticas integradas a entornos TIC, al ir más allá del análisis global exploratorio de perspectivas complementarias y alternativas, para ponerla en las prácticas pedagógicas e impactar la cultura de una pedagogía relacionada con el uso de la plataforma tecnológica, al proporcionar material contextual de episodios que iluminan similitudes, códigos detallados y corroboran espacios emergentes en la construcción del conocimiento educativo.

Por lo tanto, la colaboración trascendente virtual constituye una fuerza potencial de impacto integral flexible de las actividades que realimentan la mejora de las prácticas pedagógicas a través de experiencias tecnológicas dispuestas al servicio social para la resolución de situaciones puntuales, que a veces, no requieren la invitación de expertos. Situación que también ha sido abordada por Anthony \& Walshaw (2009) en términos de asimilar la condición trascendente de colaboración pedagógica dispuestas en las políticas públicas educativas que demandan, tanto a la institución como a los profesores, la implementación de prácticas efectivas del conocimiento colaborativo con enfoques que vinculan sus desempeños con una gama de aspectos sociales articulados al entorno, para desarrollar las capacidades del docente, la disposición de la comunidad 
y la implementación de redes pedagógicas que estimulen y orienten el sentido del discurso y la acción en la comunidad.

De modo que la situación pedagógica mediada por TIC, focaliza las respuestas necesarias en tiempos sincrónicos y asincrónicos de las actividades que los mantienen en contacto tecnológico, dentro de lo que se concibe como pedagogía tecnológica holística.

\section{Conclusiones}

- Las redes interconectadas de docentes para su formación permanente y la asimilación de nuevos escenarios pedagógicos compartidos con sus pares, constituyen un escenario potencial de autoaprendizajes, comunicación y actualización frente al compromiso del mejoramiento profesional continuo, el intercambio de información educativa y la conformación de grupos de apoyo que combinan las habilidades interpersonales, tecnológicas y la generación de competencias trasladadas a las mejores prácticas a las instituciones educativas, al llevar decisiones compartidas y alternativas de respuestas sincrónicas y asincrónicas a la orientación y manejo de situaciones complejas que requieren ser abordadas a profundidad de manera dinámica y efectiva.

- En este sentido, la asimilación trascendente de colaboración pedagógica con la utilización de las TIC abre el horizonte que involucra la acción cooperativa del equipo, donde se comparten ideas, se discuten tratamientos a los problemas comunitarios frente al desafío interdisciplinario, con enfoques más allá del aula, alientan las innovaciones de comunicación e interacción dadas en las expectativas democráticas de participación e intereses metacognitivos de pensamientos críticos que añaden experiencias y vivencias pedagógicas en la formación del docente.

\section{SUGERENCIAS}

- Las manifestaciones de cambio en el perfil de competencias tecnológicas del docente, exige el compromiso y la comprensión de los diferentes escenarios potenciales para su formación permanente en colaboración con sus pares, lo que representa un impacto implícito en los nuevos perfiles exigidos para la revisión de prácticas con el uso de las TIC, que van más allá del aula. Situación que advierte la necesidad de infraestructura, equipos y conectividad a Internet disponibles para que se promueva y apoye desde la institucionalidad, el desarrollo de este perfil colaborativo centrado en la pedagogía abordada en estos tiempos de la era digital. Por lo tanto, la sugerencia que emerge en la situación planteada, tiene que ver con la mirada holística de tales enfoques al incluir, institucionalidad, autoformación y tecnopedagogía colaborativa, en redes de docentes que desafían sus propias limitaciones epistemológicas y superan las barreras de tiempos y espacios disponibles para el aprendizaje permanente con el uso de las TIC. 


\section{Referencias Bibliográficas}

Anthony, G \& Walshaw, M. (2009). Characteristics of effective teaching of mathematics: a view from the west. Journal of Mathematics Education. 2 (2),147-164.

Asgharheidari, F \& Tahriri, A. (2015). A Survey of efl teachers' attitudes towards critical thinking instruction. Journal of Language Teaching and Research. 6 (2), 388-396. DOI: http://dx.doi.org/10.17507/jltr.0602.20

Ávalos, B \& Bascope, M. (2017). Teacher informal collaboration for professional improvement: beliefs, contexts, and experience. Education Research International. 1-13. https://doi.org/10.1155/2017/1357180

Barfield, A. (2016). Collaboration. ELT Journal. $70 \quad$ (2), 222-224. https://doi.org/10.1093/elt/ccv074.

Cleary, C \& Corti, A. (2008). L'intégration des TIC dans l'enseignement secondaire. Formation Et Pratiques D'enseignement en Questions. 7, 29-49.

Espitia, M \& Olarte, A. (2011). Explorando el valor pedagógico de los foros virtuales y los blogs para promover la colaboración y el aprendizaje: una experiencia de formación del profesorado. Colombian Applied Linguistics Journal. 13 (2), 29-42.

Geng, J., Chai, C., Jong, M\& Tsun-Hin Luk, E. (2019). Understanding the pedagogical potential of interactive spherical video-based virtual reality from the teachers' perspective through the ACE framework. Journal Interactive Learning Environments. https://doi.org/10.1080/10494820.2019.1593200.

Green, N., Wolodko, B., Stewart, C., Edwards, H., Brooks, M \& Littledyke, R. (2011). Collaborative self-study of online teaching in early childhood teacher education. International Journal for Academic Development. 18 (2), https://doi.org/10.1080/1360144X.2012.677747.

Hennessy, S., Deaney, R., \& Ruthven, K. (2015). Pedagogic strategies for using ict to support subject teaching and learning: an analysis across 15 case studies. Cambridge: University of Cambridge.

Karsentipor, T., Savoie-zajc, L \& Iarose, F. (2001). Les futurs enseignants confrontés aux TIC: changements dans l'attitude, la motivation et les pratiques pédagogiques. Education of Francophonie. XXIX. 86-124. 
Lafifi, Y \& Touil, G. (2010). Study of the impact of collaboration among teachers in a collaborative authoring system. Journal of Information Technology Education. Innovations in Practice. 9,113-132.

Mathew, P., Mathew, P \& Peechattu, P. (2017). Reflective practices: a means to teacher development. Asia Pacific Journal of Contemporary Education and Communication Technology (APJCECT). 3 (1), 126-131. ISSN: 2205-6181.

Mérida, R., González, E y Olivares, M. (2017). Networking and professional development among teachers of Early Childhood Education. Foro de Educación. 15 (23), 243-256. DOI: http://dx.doi.org/10.14516/fde.479

Mominó de la Iglesia, J \& Sigalés, C. (2017). (Cords.). El impacto de las tic en la educación. Más allá de las promesas. Barcelona: Universidad Oberta de Cataluña.

Montrieux , H., Vanderlinde, R., Schellens, T \& De Marez, L. (2015). Teaching and learning with mobile technology: a qualitative explorative study about the introduction of tablet devices in secondary education. Plos One. 10 (12). https://doi.org/10.1371/journal.pone.0144008.

Parsons, D., Thomas, H., Inkila, M., Antipas, P., Valintine, F \& Pham, T. (2015). Transforming teacher education with digital and collaborative learning and leadership. International Journal of Digital Literacy and Digital Competence. 6 (4), 30-48.

Schieffer, L. (2015). Virtual collaboration: a phenomenological study of remote online adjuncts virtual collaboration lived experiences. TOJDEL. The Online Journal of Distance Education and e-Learning. 3 (3), 90-116.

Stoll, L., Harris, A \& Handscomb, G. (2012). Great professional development which leads to great pedagogy: nine claims from research. Research and development network national themes: theme two. Escocia: National Cllege for Schol Leadership.

Strauss, A y Corbin, J. (2002). Bases de la investigación cualitativa. Técnicas y procedimientos para desarrollar la teoría fundamentada. Medellín: Universidad de Antioquia.

Treharne, G \& Riggs, D. (2015). Ensuring quality inqualitative research. In: Rohleder, P \& Lyon, A. (2014). (Edit.). Qualitative Research in Clinical and Health Psychology. 57-73. DOI: 10.1007/978-1-137-29105-9_5

Trusta, T., Krutkab, D \& Carpenterc, J. (2016). Together we are better: professional learning networks for teachers. Computers \& Education. Elsevier. 102, 15-34. https://doi.org/10.1016/j.compedu.2016.06.007 
Vartiainen, H., Nissinen, S., Pöllänen, S \& Vanninen, P. (2018). Teachers' insights into connected learning networks: emerging activities and forms of participation. American Educational Research Association.. AERA. 4 (3). https://doi.org/10.1177/2332858418799694

Voogt, J.M., Pieters, J.M \& Handelzalts, A. (2016). Teacher collaboration in curriculum design teams. Educational Research and Evaluation. 22 (3-4), 121-140. DOI: 10.1080/13803611.2016.1247725

Weitze, C. (2016). Designing pedagogical innovation for collaborating teacher teams. Journal of Education for Teaching International research and pedagogy. Cooperative Learning: Exploring Challenges, Crafting Innovations. $43 \quad$ (3), 361-373. https://doi.org/10.1080/02607476.2017.1319511

White, S. (2014). Bridging the gaps: Using social media to develop techno-pedagogical competences in pre-service language teacher education. Recherche et pratiques pédagogiques en langues de spécialité. XXXIII (2), 143-169. DOI: 10.4000/apliut.4432. 


\section{PARA CITAR EL ARTÍCULO INDEXADO.}

Zavala Morales, A., Anchundia Delgado, I., Montenegro Parrales, M. de L., Zhicay Muy, G., \& Anchundia Párraga, J. (2019). Redes interconectadas de docentes: Asimilación trascendente de colaboración $\quad$ pedagógica. $\quad$ Ciencia $\quad$ Digital, $\quad 3(3), \quad 142-161$. https://doi.org/10.33262/cienciadigital.v3i3.620

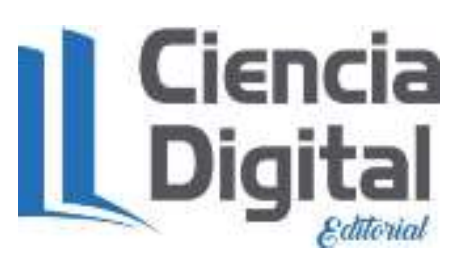

El artículo que se publica es de exclusiva responsabilidad de los autores y no necesariamente reflejan el pensamiento de la Revista Ciencia Digital.

El artículo queda en propiedad de la revista y, por tanto, su publicación parcial y/o total en otro medio tiene que ser autorizado por el director de la Revista Ciencia Digital.
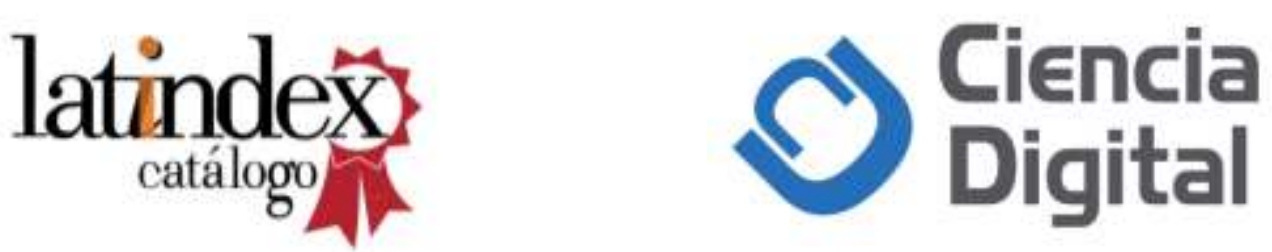